\title{
Lubiprostone: a novel treatment for chronic constipation
}

\author{
Brian E Lacy \\ L Campbell Levy
}

Section of Gastroenterology and Hepatology, Dartmouth-Hitchcock Medical Center, Lebanon NH, USA
Correspondence: Brian E Lacy

Dartmouth-Hitchcock Medical Center, Section of Gastroenterology and

Hepatology, Area 4C, I Medical Center

Drive, Lebanon, NH 03756, USA

$\mathrm{Tel}+\mathrm{I} 6036505215$

Fax +16036505225

Email brian.lacy@hitchcock.org

\begin{abstract}
Chronic constipation is highly prevalent, reduces patients' quality of life, and imposes a significant health care burden on society. Lifestyle modifications and over-the-counter agents improve symptoms of constipation in some patients, however many patients have persistent symptoms and require the use of prescription medications. Three prescription medications are currently Food and Drug Administration (FDA) approved and available for the treatment of chronic constipation in adults. This review will focus on lubiprostone, the newest medication available for the treatment of chronic constipation. Lubiprostone is a bicyclic fatty acid metabolite analogue of prostaglandin E1. It activates specific chloride channels in the gastrointestinal tract to stimulate intestinal fluid secretion, increase gastrointestinal transit, and improve symptoms of constipation. This article will provide a brief overview on chloride channel function in the gastrointestinal tract, describe the structure, function, and pharmacokinetics of lubiprostone, and discuss the safety and efficacy of this new medication.
\end{abstract}

Keywords: chloride, chloride channels, constipation, functional bowel disorders, gastrointestinal motility, intestinal secretion, irritable bowel syndrome, lubiprostone

\section{Introduction}

Constipation is a common disorder with an estimated prevalence of approximately $15 \%$ in the United States (Higgins and Johanson 2004). Women are affected more commonly than men, although the mechanism that accounts for this disparity has not been identified. Constipation is also more prevalent in the elderly, in non-Caucasians, and in patients in lower socioeconomic classes (Higgins and Johanson 2004; Lacy and Cole 2004). Although the natural history of chronic constipation is not as well studied as other common functional bowel disorders such as dyspepsia and irritable bowel syndrome (IBS), most patients with chronic constipation remain symptomatic when surveyed 18-20 months after initial evaluation (Talley et al 1992).

Although not a life-threatening illness, chronic constipation markedly affects patients' quality of life and imposes a significant economic burden to the health care system. Using the well-validated SF-36 questionnaire, several studies have shown that patients with chronic constipation note a reduction in quality of life across multiple domains, including both physical and psychological domains (Irvine et al 2002; Dennison et al 2005). The costs associated with treating chronic constipation arise due to both indirect and direct costs. Indirect costs include missing school or work (absenteeism) and being less productive at school or work (presenteeism), while the direct costs of treating constipation include office visits, diagnostic tests, and medications. Overall, it is estimated that several billion dollars are spent each year in the US treating chronic constipation (Irvine et al 2002).

The definition of constipation has evolved over the last decade and is currently based on symptoms rather than stool frequency alone. Patients with constipation often describe a constellation of symptoms that includes infrequent stools, straining, feelings 
of incomplete evacuation, and rectal or perianal fullness or discomfort. The recently released Rome III criteria have attempted to take these various symptoms into consideration (Longstreth et al 2006; see Table 1). Pathophysiologically, constipation is generally classified as either primary (eg, colonic inertia, pelvic floor dysfunction, normal transit constipation, IBS with constipation) or secondary in nature (eg, metabolic, endocrine, surgical, psychiatric) (see Table 2; Longstreth et al 2006; Brandt et al 2005).

Treating patients with constipation can be frustrating at times, because symptoms do not always accurately reflect the underlying pathophysiology nor do they predict response to treatment. Many patients initially self-treat with life-style modifications, which include drinking more water, exercising, and adding dietary fiber. Although these treatments are safe, they are usually ineffective except in patients who are fiber deficient (Young et al 1998; Muller-Lissner et al 2005). Patients with persistent symptoms then generally use over-the-counter medications, which include bulk laxatives (eg, psyllium), osmotic laxatives (eg, magnesium citrate), emollients (eg, docusate sodium), and stimulant laxatives (eg, cascara). Although some patients note an improvement in symptoms, there is little evidence documenting long-term clinical efficacy of these agents (Brandt et al 2005). Symptoms that fail to respond to this step-wise approach generally lead a patient to seek medical consultation. After an appropriate evaluation has been performed, medical therapy is usually recommended, and this may include osmotic agents (eg, polyethylene glycol, lactulose) or a chloride type 2 channel activator (eg, lubiprostone).

The efficacy of polyethylene glycol (PEG) for chronic constipation (defined as symptoms for at least six months) compared to placebo was recently studied by DiPalma and colleagues (DiPalma et al 2007). PEG relieved symptoms

Table I Rome III criteria for chronic constipation

- Symptom onset at least 6 months prior to diagnosis

- Presence of symptoms for the last 3 months (see below)

- Insufficient criteria for IBS

- Loose stools are rarely present without the use of laxatives

- Symptoms include 2 or more of the following during at least $25 \%$ of defecations:
- Straining
- Lumpy or hard stools
- Sensation of incomplete evacuation
- Sensation of anorectal obstruction or blockade
- Manual maneuvers to facilitate evacuation
- Less than 3 bowel movements per week

Modified with permission from Longstreth GF, Thompson WG, Chey WD, et al. 2006. Functional bowel disorders. Gastroenterology, 130: 1480-91. Copyright (C) 2006 Elsevier. Abbreviation: IBS, irritable bowel syndrome.
Table 2 Common causes of constipation

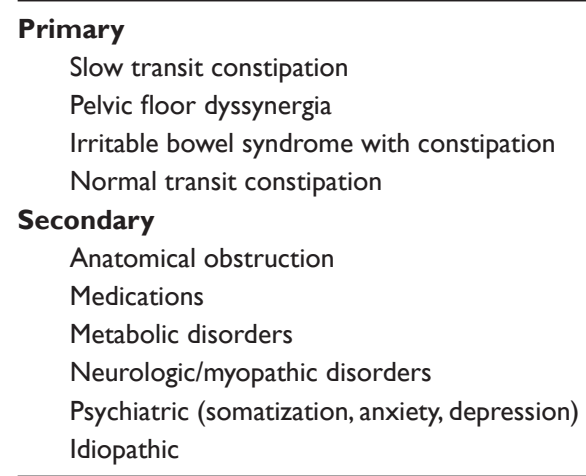

of chronic constipation (modified Rome criteria) more than half the time in $52 \%$ of subjects, while placebo was effective in $11 \%$ of subjects $(p<0.001)$. Forty percent of patients taking PEG experienced gastrointestinal side effects (abdominal distension, diarrhea, loose stools, flatulence, and nausea) compared to $25 \%$ in the placebo group $(p=0.015)$. None of these effects were individually statistically significant, and no differences in emergent adverse events were observed among elderly patients. The safety and efficacy of other osmotic agents has been discussed in two recent monographs and will not be reviewed further (Brandt et al 2005; Cash et al 2006). The remainder of this review will focus on lubiprostone.

\section{Chloride channels}

Chloride channels are found throughout the body in virtually all cell types (Chen 2005). They are pore-forming proteins that allow the transport of chloride ions across cell membranes. In the gastrointestinal (GI) tract, a number of different chloride channels play a critical role in fluid transport, and the maintenance of both cell volume and intracellular pH (Suzuki et al 2006). At present, 9 separate chloride channels (abbreviated $\mathrm{ClC}$ ) have been identified (ClC-0 - ClC7, ClC-Ka, ClC-Kb). This review, however, will focus on the $\mathrm{ClC}-2$ channel, since this chloride channel is the one selectively activated by lubiprostone.

The $\mathrm{ClC}-2$ channel is distributed throughout the GI tract, including the stomach, small intestine, and colon. It is a transmembrane protein that is highly selective for $\mathrm{Cl}$ - and is not permeable to larger anions or to cations (Mindell and Maduke 2001). ClC-2 channels are localized to the apical cell membrane in human intestine (Lipecka et al 2002).

\section{Key properties of lubiprostone Structure}

The formal chemical name of lubiprostone is difluoropentyl2-hydroxy-6-oxooctahydrocyclopenta-heptanoic acid 
(Amitiza ${ }^{\circledR}$ Package Insert). It is a white, odorless crystal and powder that is insoluble in water but soluble in ethanol. Lubiprostone is classified as a prostone, a bicyclic fatty acid compound derived from a metabolite of prostaglandin E1. Lubiprostone can tautomerize between two different forms - II is the active form (see Figure 1).

\section{Pharmacokinetics and metabolism}

The pharmacokinetic properties of lubiprostone were evaluated in a study of fasted healthy male volunteers (Amitiza ${ }^{\circledR}$ Package Insert). After ingesting a single 72 microgram dose of radio-labeled lubiprostone, $60 \%$ of the radio-label was recovered in the urine within 24 hours, and $63 \%$ was recovered in the urine by the end of one week. Thirty percent of the total administered radioactivity was recovered in the stool by one week. The parent molecule cannot be detected in plasma, urine, or stool, and it is thought that the measured radioactivity represents the active metabolite, M3. Peak plasma levels occur approximately 1.14 hours after oral administration of a single 24 microgram dose, and the halflife of lubiprostone $\left(t_{1 / 2}\right)$ has been estimated at approximately 3 hours (Amitiza ${ }^{\circledR}$ Package Insert).

Metabolism takes place within the GI tract via microsomal carbonyl reductase; the cytochrome P450 system is not involved. In contrast to the parent drug, M3 is absorbed and approximately $94 \%$ is bound to human plasma proteins. The half-life of M3 is approximately 0.9-1.4 hours. Although not tested in large studies, gender does not appear to influence the metabolism of lubiprostone. No studies have been conducted to assess the pharmacokinetic profile of lubiprostone in patients with hepatic or renal impairment. In summary, lubiprostone appears to act locally within the GI tract, has a fairly quick onset of action, is rapidly metabolized on the cell surface, and thus should have few drug-drug interactions.

\section{Mechanism of action}

Lubiprostone specifically activates $\mathrm{ClC}-2$ channels on the apical membrane of epithelial cells (Cuppoletti et al 2004). Highly specific inhibitors of $\mathrm{ClC}-2$ channels are not available, and thus appropriate blocking experiments cannot be performed. Activation of $\mathrm{ClC}-2$ channels causes an efflux of chloride into the lumen of the GI tract, followed by an efflux of sodium ions in order to maintain isoelectric neutrality. It should be noted that sodium efflux does not occur through the apical membrane but rather occurs through a paracellular pathway. Water then follows along the paracellular pathway in order to maintain isotonic equilibrium. Animal studies have demonstrated that administration of lubiprostone increased intestinal fluid secretion in a dose-dependent manner compared to vehicle (Ueno et al 2004). Animal studies have also demonstrated that lubiprostone does not appear to have a direct stimulatory effect on gastrointestinal smooth muscle (Perentesis et al 2005).

In summary, lubiprostone stimulates $\mathrm{ClC}-2$ channels and promotes intestinal fluid secretion. The secretion of fluids into the GI tract adds fluid to stool and promotes increased transit, likely through stimulation of local receptors sensitive to stretch and distention (Grider and Jin 1994; Camilleri et al 2006). Lubiprostone does not appear to have any significant activity on smooth muscle or on prostaglandin receptors.

\section{Effects on reproduction and development}

In animal models, lubiprostone does not appear to have direct teratogenic effects (Amitiza ${ }^{\circledR}$ Package Insert; Crawford et al 2005). However, administration of lubiprostone at 6-10 times the recommended level was associated with an increased rate of fetal loss in a guinea pig model. This was not felt to be a direct effect, but rather was thought to occur secondary to weight loss in the pregnant animals (Amitiza ${ }^{\circledR}$ Package Insert; Crawford et al 2005). Fetal loss did not occur in similarly

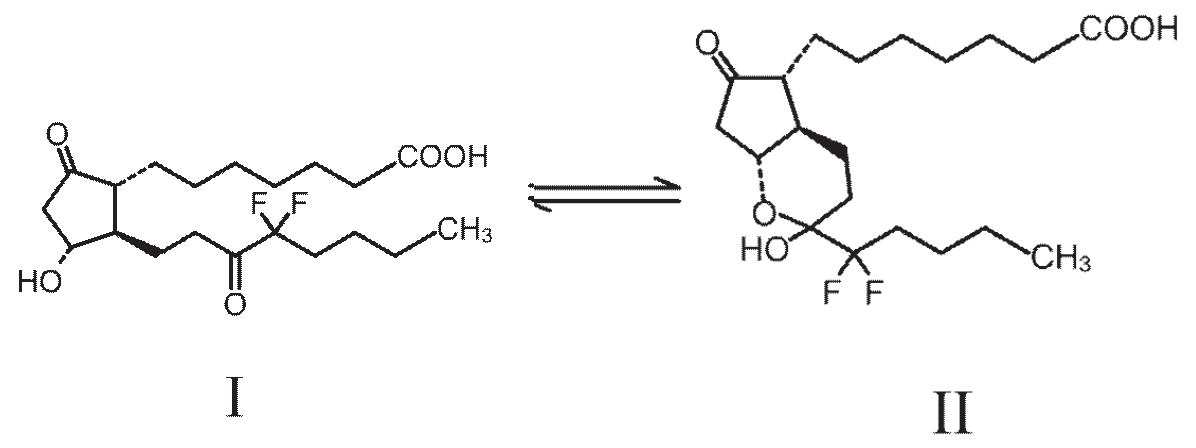

Figure I Structure of lubiprostone. 
Table 3 Key properties of lubiprostone

\begin{tabular}{ll}
\hline Recommended dosage & $24 \mu \mathrm{g}$ twice daily \\
Route of administration & Oral \\
Time to peak plasma concentration & Approximately I.5 hours \\
Estimated terminal half-life & Approximately 3 hours \\
Bioavailability & $<1 \%$ \\
Metabolite & M3 - partially active \\
Metabolism & Carbonyl reductase \\
Pregnancy category & $\mathrm{C}$ \\
\hline
\end{tabular}

designed experiments involving mice and rats. Due to the adverse effects observed in the guinea pig model and the lack of well-controlled studies in pregnant women, lubiprostone is accordingly rated a pregnancy category " $\mathrm{C}$ " by the FDA.

\section{Clinical studies}

The efficacy of lubiprostone for the treatment of constipation has been studied in a recently published double-blind, placebo-controlled dose ranging study, two separate phase 3 trials, a withdrawal study, and several larger open-label studies.

\section{Randomized controlled trials}

The dose-ranging study to evaluate the efficacy and safety of lubiprostone involved 129 subjects with symptoms of chronic (at least 6 months) constipation (Johanson and Ueno 2007). Constipation was defined as fewer than 3 spontaneous bowel movements (SBM) per week, together with one or more of the following symptoms occurring at least $25 \%$ of the time: hard stools, straining, or feelings of incomplete evacuation. The subjects received either lubiprostone $(24,48$, or $72 \mu \mathrm{g} /$ day $)$ or placebo for 3 consecutive weeks after a 2 -week washout period. The patients were predominantly female ( $\geq 84 \%$ in each treatment arm) and Caucasian ( $\geq 81 \%$ in each treatment arm). The mean age was 48.3 years with $10 \%$ of patients being at least 65 years of age. Patients in the treatment groups had a significant increase in the average number of weekly SBMs during the first $(p=0.006)$ and second week of the study $(p=0.014)$, and over the entire study period $(p=0.046)$ when compared to placebo. Each of the three treatment arms showed significant improvement in SBM frequency at week 2 of the study compared with placebo ( $p \leq 0.020$ ), but only the $48 \mu \mathrm{g}$ treatment group showed a statistically significant improvement over the entire treatment period $(p=0.015)$. A larger percentage of patients in each treatment arm had a SBM within 24 hours of initial dosing of lubiprostone compared to placebo; the difference for the two highest dosing groups was statistically significant (placebo group $=27.3 \%$;
$24 \mu \mathrm{g}$ group $=44.8 \%, 48 \mu \mathrm{g}=59.4 \% ;(\mathrm{p}=0.009)$; $72 \mu \mathrm{g}=63.6 \% ; \mathrm{p}=0.003)$. Several measures of constipation were assessed each week during the trial period using 4 and 5 point scales. The lubiprostone treatment group experienced a statistically significant improvement over the entire treatment period when compared to baseline in the mean degree of straining ( $p<0.005)$, stool consistency ( $p<0.0001)$, abdominal bloating $(\mathrm{p}=0.035)$, severity of constipation $(p=0.010)$, and overall rating of treatment effectiveness $(p=0.045)$. Abdominal discomfort also improved but did not reach statistical significance $(\mathrm{p}=0.136)$.

There were no drug-related serious adverse events during the trial. Clinical assessments including hematology, blood chemistries, vital signs, physical examination, and electrocardiogram (ECG) showed no clinically significant trends during the study. One patient in each treatment group had a QT change of 30-60 seconds on ECG from baseline, which was considered non-specific. Sixty-nine percent of patients in the lubiprostone group experienced at least one adverse event compared to $39 \%$ of patients taking placebo. Adverse events of severe intensity were reported by $12(9 \%)$ patients. Of these events, 10 were experienced by more than 1 patient and included: headache ( 2 patients in the $72 \mu \mathrm{g}$ arm), diarrhea (4 patients in the $72 \mu \mathrm{g}$ arm), abdominal pain (1 patient in the placebo arm and 1 patient in the $48 \mu \mathrm{g}$ group), and nausea (1 patient in each of the lubiprostone $48 \mu \mathrm{g}$ and $72 \mu \mathrm{g}$ groups). Nausea was the most common adverse event. No patients taking placebo reported nausea, but it was reported by $17 \%$ of the $24 \mu \mathrm{g}$ group, $43.8 \%$ of the $48 \mu \mathrm{g}$, and $36.4 \%$ of the $72 \mu \mathrm{g}$ group. Nausea in each of the patients except one (30/31) was judged to be related to the medication by the investigator. Four percent of study patients discontinued the study because of nausea. The mechanism underlying the development of nausea in patients treated with lubiprostone is not known.

Data from two similar phase III multi-center trials reinforce the findings of the initial dose-ranging study (Johanson et al 2005a; Johanson et al 2008). Inclusion criteria required that patients have symptoms of chronic constipation as previously described (Johanson and Ueno 2007). One trial involved 242 subjects (mean age $=48.6$ years; $90 \%$ women; $13 \% \geq 65$ years age) from 20 centers across the US (Johanson et al 2008). After a 2-week baseline period, lubiprostone $(24 \mu \mathrm{g})$ or placebo was administered twice daily for 4 weeks. Patients were prohibited from using prescription or over-the-counter constipation remedies during the washout and study periods. Dulcolax ${ }^{\circledR}$ suppositories or Fleet ${ }^{\mathbb{B}}$ enemas were authorized by the study investigators as "rescue" therapy for those subjects without 
a BM for 3 or more consecutive days. Compared to placebo, the treatment group had more SBMs during week 1 (5.69 vs $3.46, p=0.0001$ ), and the effect was sustained during each of the subsequent weeks of the study. A larger percentage of patients on lubiprostone had an SBM within 24 hours $(56.7 \%$ vs $36.9 \%, p=0.0024)$ and within 48 hours $(80.0 \%$ vs $60.7 \%$, $\mathrm{p}=0.0013)$. The need for rescue medications was similar in both groups at baseline but decreased in the lubiprostone group by the end of the study period $(35.6 \%$ vs $50.8 \%$, $\mathrm{p}=0.0357)$. Symptom scores were significantly improved with lubiprostone compared to placebo for weeks $1-4$ for stool consistency ( $p<0.0001)$, straining ( $\mathrm{p} \leq 0.0001)$, and constipation severity ( $\mathrm{p} \leq 0.0003$ ). Abdominal bloating was improved in the lubiprostone treated group compared to placebo during weeks $1-2$ ( $\mathrm{p} \leq 0.031$ ), while abdominal discomfort scores were significantly improved for weeks $2-4$ ( $\mathrm{p} \leq 0.045)$. Seventy percent of subjects on lubiprostone reported at least one adverse event compared to $50.8 \%$ of patients on lubiprostone $(p=0.0026)$. Six percent of events were severe, but there was no significant difference in incidence between treatment groups. The most common treatment-related adverse event was nausea, occurring in $31.7 \%$ of the lubiprostone group and $3.3 \%$ of the placebo group ( $p<0.001)$. Five percent of patients discontinued the study due to nausea.

The second phase III trial included 237 subjects (mean age $=45.8$ years; $88 \%$ women) (Johanson et al 2005a) Patients on lubiprostone experienced significant improvement in the frequency of weekly bowel movements (5.89 vs $3.99, \mathrm{p}<0.0001)$. Similar to the previously described studies, patients on lubiprostone reported improvements in subjective measures of constipation, and more patients experienced a SBM within the first 24 hours in the lubiprostone group than the placebo group (61\% vs $31 \%$, p $<0.0001)$. Nausea, headache, and diarrhea were again the most commonly reported adverse effects. Mild to moderate nausea occurred more frequently in the lubiprostone treated group than in the placebo group in the second study ( $21 \%$ vs $4.2 \%$; p value not reported) (Johanson et al 2005b). No serious adverse events were reported; 15 lubiprostone patients withdrew from the second trial.

The potential for rebound constipation developing following drug withdrawal (after 4 weeks of lubiprostone treatment) was evaluated in 128 subjects (mean age and gender not reported) with chronic constipation, as previously defined (Johanson et al 2004). Lubiprostone (24 $\mu \mathrm{g}$ ) was administered twice daily for 4 weeks after a 2 -week washout period. Subjects were then randomized to receive
3 additional weeks of lubiprostone or placebo. A rapid and sustained improvement in SBM similar to that seen in the other trials was observed (1.36 per week at baseline vs 6.25 , 5.94, and 5.52 at weeks 1,2, and 3. respectively; $\mathrm{p}<0.0001$ at all weeks). Three weeks after randomization to placebo or to continued lubiprostone, the SBM frequency declined in the placebo group (3.04 vs 5.59; p = 0.0464) but remained improved compared to baseline (3.04 vs 1.36; $\mathrm{p}=0.0223$ ), arguing against significant rebound constipation after lubiprostone withdrawal.

\section{Open-label studies}

Three large, open-label studies investigated the long-term efficacy of lubiprostone using five point scales as a subjective measure of constipation. The severity of constipation, bloating, and abdominal discomfort were periodically assessed among 880 patients (308 patients were entered into one 24-week study; a total of 572 patients were entered into 2 separate 48-week trials) (Johanson et al 2006a). There were statistically significant improvements in all symptoms throughout the trial periods $(\mathrm{p}<0.0001)$. Assessments of constipation severity improved across all three studies by an average of $26 \%$ at weeks $4-6(n=28), 29 \%$ at week 24 $(\mathrm{n}=512)$, and $28 \%$ at week $48(\mathrm{n}=281)$. Bloating improved by an average of $18 \%$ at weeks $4-6(n=829)$, and $20 \%$ at both 24 weeks $(n=512)$ and 48 weeks $(n=282)$. Abdominal discomfort improved by an average of $15 \%$ at week 1 $(\mathrm{n}=619), 18 \%$ at 24 weeks $(\mathrm{n}=512)$, and $17 \%$ at 48 weeks $(\mathrm{n}=282)$.

Women comprised the majority of subjects in the initial trials of lubiprostone. A subgroup analysis of the four-week controlled trials was performed to evaluate efficacy in men (Ueno et al 2006a). Pooled data yielded 27 male placebo subjects and 32 lubiprostone subjects. Male subjects taking lubiprostone experienced 5.69-6.05 SBMs per week compared to $2.55-3.23$ in the placebo group $(\mathrm{p}<0.0489$ at week $3, p=0.0503$ at week 4$)$. These rates were higher compared to females in the trials (4.99-5.75 SBM/week). Fifty percent of male subjects taking lubiprostone experienced at least one side effect compared to $33.3 \%$ of men taking placebo (details of adverse effects were not reported).

\section{Lubiprostone use in elderly patients}

Analysis of the clinical trials performed to date provides insight into the safety and efficacy of lubiprostone in elderly subjects (defined as $>65$ years old). Pooled data from 3 of the previously described 4-week controlled trials yielded 31 elderly patients in the placebo groups and 26 patients in 
the lubiprostone groups (Ueno et al 2006b). Improvements in SBM per week, during each of the 4 weeks, ranged from 4.6-5.4 in the lubiprostone treated group compared to $1.29-2.27$ for those treated with placebo $(p<0.0286)$. Fewer adverse events were reported in elderly subjects treated with lubiprostone compared to subjects treated with placebo $(46 \%$ vs $61 \%$; p value not reported). In a subgroup analysis of the open-label trials, pooled data yielded 163 elderly subjects ( $\geq 65$ years old) and 715 non-elderly subjects (Ueno et al 2006c). Fewer elderly patients reported adverse effects when treated with lubiprostone compared to non-elderly subjects ( $74.2 \%$ vs $80.1 \%$; $p$ value not reported). The most common side effect, nausea, was less common among elderly subjects than younger patients (17.8\% vs $29.4 \%$; $p$ value not reported). In summary, despite the smaller total number of elderly patients, analysis of pooled data indicates that lubiprostone is both effective and safe in the treatment of constipation in the elderly.

\section{Adverse effects and safety}

One study of 26 healthy volunteers was designed to specifically evaluate the safety and tolerability of lubiprostone (Ueno 2005). Subjects in each group received either placebo or one of three doses of lubiprostone $(72 \mu \mathrm{g}, 90 \mu \mathrm{g}$, or $108 \mu \mathrm{g}$ divided into 3 daily doses). The subjects were monitored closely for 7 days without any changes in electrocardiograms, laboratory testing, or vital signs. There were no serious adverse events. Two subjects who withdrew from the study did so voluntarily and had no adverse events. Almost all of the adverse events consisted of gastrointestinal symptoms including vomiting, nausea, and abdominal cramping. As expected, subjects receiving lubiprostone experienced more diarrhea than those on placebo. The effect did not seem to be dose dependent.

Pooled data from previously mentioned studies, including 258 patients from 2 blinded studies and 858 patients from 3 open-label trials, were reviewed for a change in serum electrolytes due to lubiprostone (24 $\mu \mathrm{g}$ bid) (Rivera et al 2007). In the blinded and open-label trials, $10.5 \%$ and $18.6 \%$ of patients respectively were 65 years of age or greater. There were no changes in electrolytes observed over the treatment period, which ranged from 12 to 48 weeks.

ECG changes due to lubiprostone were evaluated in 177 patients with chronic constipation and 68 healthy male and female volunteers (age was not reported) (Sprenger et al 2007). ECGs before and after a single dose of $24 \mu \mathrm{g}$ lubiprostone or a supratherapeutic dose of $144 \mu \mathrm{g}$ did not show any changes.
We do not recommend using lubiprostone during pregnancy due to the lack of human reproductive data, and the manufacturers have recommended using lubiprostone during pregnancy only if the benefits significantly outweigh potential risks. Prior to initiating therapy, a pregnancy test should be checked in all women of child-bearing age not using a reliable form of birth control. Given the limited data available, we also do not recommend using lubiprostone in women who are breastfeeding.

\section{Other potential uses Irritable bowel syndrome with constipation}

The results of the first phase III study evaluating the safety and efficacy of lubiprostone in patients with IBS and constipation were recently published in abstract form (Drossman et al 2007). Earlier phase II dose-ranging studies had determined that $8 \mathrm{mcg}$ of lubiprostone twice daily improved IBS symptoms (Johanson et al 2006b). However, the sample size was quite small. In the current study 1171 adults diagnosed with IBS-C using the Rome II criteria were randomized to receive either 12 weeks of twice daily lubiprostone $(8 \mu \mathrm{g})$ or placebo. Most patients were women (91.6\%), and most were between the ages of 18 and $65(91.7 \%)$. The primary efficacy variable was a global question rating overall IBS symptoms, while a 7-point balanced scale was used to rate changes in individual symptoms. Patients reporting at least moderate relief for 4 out of 4 weeks, or patients reporting significant relief 2 out of 4 weeks were considered monthly responders, and patients had to be a monthly responder for at least 2 out of the 3 months in order to qualify as an overall responder. The authors reported that patients receiving lubiprostone were nearly twice as likely as those receiving placebo to achieve overall response $(17.9 \%$ vs $10.1 \% ; \mathrm{p}=0.001)$. Secondary endpoints, including abdominal pain, bloating, straining, stool consistency, and constipation were all significantly improved in the lubiprostone group compared to the placebo group ( $p$ $<0.05$ for all endpoints). Lubiprostone was generally well tolerated. The most common treatment related side effects were nausea ( $8 \%$ vs $4 \%$ in placebo) and diarrhea ( $6 \%$ vs $4 \%$ in placebo). Of note, it is thought that the low placebo rate in this study is due to the much stricter standards for determining whether a patient is classified as a responder. Also, the lower dose $(8 \mu \mathrm{g})$ was chosen based on prior dose-ranging studies and may reflect the fact that patients with IBS often respond to medications at lower doses than other patients. Further trials are planned to assess the long-term benefits of lubiprostone in patients with IBS and constipation. 


\section{Opioid-induced constipation}

Constipation is a frequent side effect of opioid use. Studies to evaluate the efficacy and safety of lubiprostone in opioidinduced constipation in humans have begun, although data are not yet available. Lubiprostone does appear to improve opioid-induced constipation in a mouse model, however (Ueno et al 2006d).

\section{Summary}

Chronic constipation is a highly prevalent disorder that is frequently resistant to treatment with lifestyle modifications and over-the-counter remedies. Treatment options with prescription medications are limited, especially given the recent withdrawal of tegaserod from the marketplace (Zelnorm 2007). Lubiprostone, a new class of medication approved for the treatment of chronic constipation in both men and women (age 18 and over), was approved by the FDA on January 31, 2006 and is marketed under the trade name Amitiza ${ }^{\circledR}$. It is available in a gelatin capsule and the recommended dosing is $24 \mu \mathrm{g}$ p.o. twice daily. No restrictions have been placed on the length of its use.

Lubiprostone selectively stimulates type 2 chloride channels in epithelial cells thereby causing an efflux of chloride into the intestinal lumen. The resultant fluid secretion into the gastrointestinal lumen provides a bolus effect that softens stool, increases intestinal transit, and improves symptoms of constipation. Lubiprostone acts locally within the intestinal tract, is rapidly metabolized, and has very low systemic bioavailability.

Preclinical trials have shown high specificity of the drug for ClC-2 channels. Animal studies have shown that lubiprostone significantly increases small intestinal fluid volume and also elevates intestinal fluid chloride concentration without altering serum electrolyte concentrations. Doubleblinded, randomized human studies have demonstrated that lubiprostone accelerates small bowel and colonic transit. Well-designed clinical trials and larger open-label trials have established lubiprostone as a safe and effective treatment option for chronic constipation that is generally welltolerated. Future studies will determine its utility in other functional bowel disorders, especially opioid-induced constipation and irritable bowel syndrome with constipation.

\section{References}

Amitiza ${ }^{\circledR}$ Package Insert.

Brandt LJ, Prather CM, Quigley EMM, et al. 2005. Systematic review on the management of chronic constipation in North America. Am J Gastroenterol, 100:S5-S22.

Camilleri M, Bharucha AE, Ueno R, et al. 2006. Effect of a selective chloride channel activator, lubiprostone, on gastrointestinal transit, gastric sensory, and motor functions in healthy volunteers. Am JPhysiol Gastrointest Liver Physiol, 290:G942-G7.
Cash BD, Lacy BE. 2006. Systematic review: FDA-approved prescription medications for adults with constipation. Gastroenterol Hepatol, 2:736-49.

Chen TY. 2005. Structure and function of ClC channels. Annu Rev Physiol, 67:809-39.

Crawford DF, Perentesis GP, Engelke KJ, et al. 2005. Effects of lubiprostone, a novel GI chloride channel activator, on reproductive and developmental toxicity endpoints in rats. Am J Gastroenterol, 100:S331.

Cuppoletti J, Malinowska DH, Tewari KP, et al. 2004. SPI-0211 activates T84 cell chloride transport and recombinant human $\mathrm{ClC}-2$ chloride currents. Am J Physiol Cell Physiol, 287:C1173-C83.

Dennison C, Prasad M, Lloyd A, et al. 2005. The health-related quality of life and economic burden of constipation. Pharmacoeconomics, 23:461-7.

DiPalma JA, Cleveland MV, McGowan J, et al. 2007. A randomized, multicenter, placebo-controlled trial of polyethylene glycol laxative for chronic treatment of chronic constipation. Am J Gastroenterol, 102:1436-41.

Drossman DA, Chey WD, Panas R, et al. 2007. Lubiprostone significantly improves symptom relief rates in adults with irritable bowel syndrome and constipation (IBS-C): data from two, twelve-week, randomized, placebo-controlled, double blind trials. Gastroenterol, 132:2586-7.

Grider JR, Jin JG. 1994. Distinct populations of sensory neurons mediate the peristaltic reflex elicited by muscle stretch and mucosal stimulation. J Neurosci, 14: 2854-60.

Higgins PDR, Johanson JF. 2004. Epidemiology of chronic constipation in North America: A systematic review. Am J Gastroenterol, 99:750-9.

Irvine EJ, Ferrazzi S, Pare P, et al. 2002. Health-related quality of life in functional GI disorders: focus on constipation and resource utilization. Am J Gastroenterol, 97:1986-93.

Johanson JF, Gargano MA, Holland PC, et al. 2004. Phase III, randomized withdrawal study of RU-0211, a novel chloride channel activator for the treatment of constipation. Gastroenterol, 126:A298. Abstract 749.

Johanson JF, Gargano MA, Holland PC, et al. 2005a. Initial and sustained effects of lubiprostone, a chloride channel-2 (ClC-2) activator for the treatment of constipation: data from a 4-week Phase III study. Am J Gastroenterol, 100:A884.

Johanson JF, Gargano MA, Holland PC, et al. 2005b. Phase III study of lubiprostone, a chloride channel-2 (ClC-2) activator for the treatment of constipation: safety and primacy efficacy. Am J Gastroenterol, 100:A896.

Johanson JF, Morton D, Geenan J, et al. 2008. Multicenter, 4-week, double-blind, randomized, placebo-controlled trial of lubiprostone, a locally-acting type- 2 chloride channel activator, in patients with chronic constipation. Am J Gastroenterol, 103:170-7.

Johanson JF, Panas R, Holland PC, et al. 2006a. Long-term efficacy of lubiprostone for the treatment of chronic constipation. Gastroenterol, 130:A317.

Johanson JF, Panas R, Holland PC, et al. 2006b. A dose-ranging, doubleblind, placebo-controlled study of lubiprostone in subjects with irritable bowel syndrome and constipation (IBS-C). Gastroenterol, 130:A25.

Johanson JF, Ueno R. 2007. Lubiprostone, a locally acting chloride channel activator, in adult patients with chronic constipation: a double-blind, placebo-controlled, dose-ranging study to evaluate efficacy and safety. Aliment Pharmacol Ther, 25:1351-61.

Lacy BE, Cole MS. 2004. Constipation in the older adult. Clinical Geriatr, 12:44-54.

Lipecka J, Bali M, Thomas A, et al. 2002. Distribution of ClC-2 chloride channel in rat and human epithelial tissues. Am J Physiol Cell Physiol, 282:C805-C16.

Longstreth GF, Thompson WG, Chey WD, et al. 2006. Functional bowel disorders. Gastroenterology, 130:1480-91.

Mindell JA, Maduke M. 2001. ClC chloride channels. Genome Bio, 2:3003.1-3003.6.

Muller-Lissner SA, Kamm MA, Scarpignato C, et al. 2005. Myths and misconceptions about chronic constipation. Am J Gastroenterol, 100:232-42. 
Perentesis GP, Crawford DF, Engelke KJ, et al. 2005. Effects of lubiprostone, a novel GI chloride channel activator, on isolated smooth muscle. Am J Gastroenterol, 100:A900.

Rivera E, Wahle A, Joswick TR, et al. 2007. Lubiprostone, a novel type-2 chloride channel (Clc-2) activator, does not affect serum electrolyte balance in elderly and non-elderly patients with chronic idiopathic constipation. Gastroenterol, 132:A191-2.

Sprenger C, Copa A, Morganroth J, et al. 2007. Effect of lubiprostone, a unique agent for the treatment of chronic idiopathic constipation, on clinical electrocardiogram results. Gastroenterol, 132:A325.

Suzuki M, Morita T, Iwamoto T. 2006. Diversity of Cl channels. Cell Mol Life Sci, 63:12-24.

Talley NJ, Weaver AL, Zinsmeister AR, et al. 1992. Onset and disappearance of gastrointestinal symptoms and functional gastrointestinal disorders. Am J Epidemiol, 136:165-77.

Ueno R. 2005. Multiple, escalating, oral-dose study to assess the safety, tolerance, and pharmacodynamic profile of lubiprostone in normal healthy volunteers. Neurogastroenterol Motil, 17:626.

Ueno R, Hiroyoshi O, Engelke KJ. 2006d. Effect of lubiprostone on morphineinduced constipation and analgesia. Gastroenterol, 130:A373-4.
Ueno R, Joswick TR, Wahle A, et al. 2006a. Efficacy and safety of lubiprostone for the treatment of chronic constipation in male vs. female subjects. Gastroenterol, 130:A322.

Ueno R, Joswick TR, Wahle A, et al. 2006b. Efficacy and safety of lubiprostone for the treatment of chronic constipation in elderly vs. non- elderly subjects. Gastroenterol, 130:A189.

Ueno R, Osama H, Habe T, et al. 2004. Oral SPI-0211 increases intestinal fluid secretion and chloride concentration without altering serum electrolyte levels. Gastroenterol, 126:A298.

Ueno R, Panas R, Wahle A, et al. 2006c. Long-term efficacy and safety of lubiprostone for the treatment of chronic constipation in elderly subjects. Gastroenterol, 130:A188.

Young RJ, Beerman LE, Vanderhoof JA. 1998. Increasing oral fluids in chronic constipation in children. Gastroenterol Nurs, 21:156-61.

Zelnorm (tegaserod maleate) Information. 2007. FDA/Center for Drug Evaluation and Research. Accessed September 27, 2007. URL: www. fda.gov/CDER/Drug/infopage/zelnorm/default.htm. 